\title{
A Thermoresponsive Three-Dimensional Fibrous Cell Culture Platform for Enzyme-Free Expansion of Mammalian Cells
}

Afnan M. Aladdad a, Mahetab H. Amer a, Laura Sidney ${ }^{b}$, Andrew Hopkinson ${ }^{b}$, Lisa J. White ${ }^{a}$, Cameron Alexandera and Felicity R. A. J. Rose ${ }^{\text {** }}$

aSchool of Pharmacy, University of Nottingham, Nottingham, NG72RD, UK.

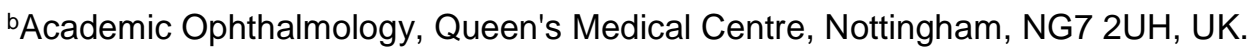

${ }^{*}$ Corresponding author: felicity.rose@nottingham.ac.uk. 


\title{
A Thermoresponsive Three-Dimensional Fibrous Cell Culture Platform for Enzyme-Free Expansion of Mammalian Cells
}

\begin{abstract}
A three-dimensional thermoresponsive fibrous scaffold system for the subsequent extended culture and enzyme-free passaging of a range of mammalian cell types is presented. Poly(PEGMA $\left.{ }_{188}\right)$ was incorporated with poly(ethylene terephthalate) (PET) via blend-electrospinning to render the fibre thermoresponsive. Using primary human corneal stromal stem cells as an therapeutically relevant exemplar, cell adhesion, viability, proliferation and phenotype on this fibrous culture system over numerous thermal enzyme-free passages is described. We also illustrate the versatility of this system with respect to fabricating thermoresponsive fibres from biodegradable polymers and for the culture of diverse mammalian cell types including mesenchymal stem cells, colon adenocarcinoma cells, and $\mathrm{NIH}$ 3T3 fibroblasts. This thermoresponsive scaffold system combines the advantages of providing a physiologically relevant environment to maintain a desirable cell phenotype, allowing routine enzyme-free passaging and expansion of cultured cells, whilst offering mechanical support for cell growth. The system described in this study presents a versatile platform for biomedical applications and more specfically for the expansion of mammalian cells destined for the clinic.
\end{abstract}

Keywords: 3D cell culture, corneal stem cells, electrospinning, thermoresponsive, tissue engineering

\section{Introduction}

The application of cells within a clinical setting is dependent on meeting regulatory requirements and developing techniques that are approved by the regulatory authorities remains a challenge. Such techniques include optimal culture systems for the expansion of cell populations whilst maintaining a desired cell phenotype and without the use of exogenous factors that may pose a risk of contamination. Conventional cell passaging requires the use of proteolytic enzymes to digest cell membrane proteins, primarily integrins, which are responsible for cell-cell and cell-substrate interactions. This process can affect cell adhesion to further substrates, impact proliferative ability, and influence cell metabolism and quality.[1-3] There is a pressing need to develop alternative culture environments that do not require the use of proteolytic enzymes. 
Synthetic 'smart' biomaterials, on which the bio-adhesive properties of the surface can be modulated by external stimuli without the need for enzyme treatment, are therefore of interest. By utilising the thermallymodulated properties of these surfaces, cells can be cultured at temperatures above the lower critical solution temperature (LCST) of the thermoresponsive polymer (hydrophobic surface), then harvested by lowering the culture temperature below the LCST (hydrophilic surface).[4] Such a system eliminates the need for proteolytic enzymes.

Two-dimensional (2D) thermoresponsive substrates have been studied for applications in cell sheet engineering $[1,5,6]$ and as tuneable artificial extracellular matrices for different cell types, such as hMSCs. [7] However, the lack of a 3D culture environment significantly impacts cell morphology, proliferation and phenotype.[8-10] Moreover, difficulties in using enzymatic treatment to extract cells from 3D culture systems highlight the need for thermoresponsive scaffolds for long-term culture and passaging of clinically-relevant cell types. Electrospinning is an efficient method for the fabrication of nano- or micro-fibrous scaffolds with controlled morphology, porosity and composition.[11] PEG-based polymers are widely used for drug delivery and biological applications, and possess a reversible phase transition.[2, 12] In contrast, PNIPAAm-based polymers possess both strong hydrogen bond donors as well as $\mathrm{H}$-bond acceptors in their structure, which can lead to irreversible inter-and intra-chain associations and entanglement.[13] This makes switchable PEG-based substrates for cell culture applications desirable, as the process of cell attachment/detachment is unhindered by irreversible dehydration.[13]

This study presents novel 3D thermoresponsive co-electrospun fibrous materials that can be used for the extended maintenance and enzyme-free passaging of anchorage-dependent mammalian cells at physiological conditions. This 3D system is versatile due to the thermoresponsive fibres being generated from polymer blends and is therefore amenable to any polymer being blended with the thermoresponsive component. These fibres present a 3D environment that supports cell growth in addition to mild and efficient cell harvesting by cooling. Since tissue regeneration in vivo is characterised by dynamic attachment/detachment of various cell populations, this study can also inform the design of advanced scaffolds for tissue engineering. Comprehensive cell culture and passaging experiments were performed using primary human corneal stromal stem cells (hCSSCs) to investigate the effects of extended culture and seven enzyme-free thermal passages on the phenotype of a therapeutically relevant cell phenotype. Extended two-dimensional culture of hCSSCs in vitro results in their dedifferentiation from a quiescent, 
dendritic cell phenotype into an undesirable fibroblastic/myofibroblast repair phenotype, which in vivo leads to scarring and blindness.[14-16] Three-dimensional culture environments have been reported to promote the reversion of activated corneal stromal cells to a quiescent keratocyte phenotype.[16,17,36] Diverse exemplar cell types, such as mesenchymal stem cells (ihMSCs; stem cell type), colon adenocarcinoma cells (Caco2; carcinoma cell type) and NIH-3T3 fibroblasts (differentiated cell type) were used to illustrate the biocompatibility of these electrospun substrates across different mammalian cell types whilst confirming that the thermoresponsive phenomenon is not cell line-specific. Cells were evaluated for adhesion, viability, and subsequent proliferation on the scaffolds over numerous thermal enzyme-free passages. Electrospun thermoresponsive fibres offers a robust cell culture platform for a range of potential applications, including large-scale cell expansion and 3D culture systems that allow dynamic control over the cell adhesive environment.

\section{Materials and methods}

\subsection{Materials}

Poly $($ PEGMA 188$)$ was prepared by free radical polymerization following a published method [18] as detailed in Supporting Information (section 1.1; $\mathrm{Mw}$ [gel permeation chromatography [GPC] $=50.8 \mathrm{kDa}, \bigoplus=1.8$ ). While commercial PET contains stabilisers, such as glass, PET from drinks bottles is food-grade and contains only PET, making it a purer source of PET. PET collected from commercial grade drinks bottles (bottles were ground and pooled into a single batch and stored at $-20^{\circ} \mathrm{C}$ until required). NMR spectra $\left({ }^{1} \mathrm{H}\right.$ and ${ }^{13} \mathrm{C}$ ) showed the expected resonances with no impurities above the instrumental level of detection; Figure S2) and PLGA (lactide/glycolide ratio $75: 25, \mathrm{Mn}[\mathrm{GPC}]=68 \mathrm{kDa}, Ð=1.7$ ) purchased from SigmaAldrich were used. Solvents were HPLC grade (Sigma-Aldrich) and used without further purification.

\subsection{Polymer solutions and electrospinning}

PET polymer $(30 \% \mathrm{w} / \mathrm{v})$ was dissolved in a mixture of $3 \mathrm{~mL}$ of TFA/3 $\mathrm{mL}$ of DCM, and PLGA was dissolved in $6 \mathrm{~mL}$ of DCM. For co-polymer solutions, $4 \%(w / v)$ of the thermoresponsive poly $\left(\mathrm{PEGMA}_{188}\right)$ was added to the PET or PLGA polymer solutions. A 4\% $(w / v)$ poly $\left(\mathrm{PEGMA}_{188}\right)$ polymer was then added to the mixture. The same concentration of poly $\left(\right.$ PEGMA $\left._{188}\right)$ was prepared with $18.5 \%(w / v)$ PLGA polymer (Sigma-Aldrich, lactide/glycolide ratio 75/25, $\mathrm{Mn}$ [gel permeation chromatography, GPC] $=68 \mathrm{kDa}, Ð=1.7$ ) in $6 \mathrm{~mL}$ of $\mathrm{DCM}$. 
Mixtures were stirred at RT for $24 \mathrm{~h}$ to ensure complete solvation. Three different batches were prepared for each concentration on different days.

For electrospinning, $6 \mathrm{~mL}$ of the polymer solution prepared were taken into a $10 \mathrm{~mL}$ plastic syringe fitted with an 18-gauge blunt needle (Figure 1). The loaded syringe was then mounted onto a syringe pump (Harvard) and the polymer solution was pumped through the needle at different rates until regular dripping occurred. A voltage was then applied to the needle tip (positive electrode) using a high-voltage unit (Glassman High Voltage Inc.). The solution was spun onto a rectangular stainless-steel collector plate $\left(6 \mathrm{~cm}^{2}\right)$, which was grounded via an earthed electrode. The working distance between the needle and the collector plate was set to approximately $15 \mathrm{~cm}$. The entire polymer solution was spun (parameters detailed in Table 1), and the plate was rotated at $90^{\circ}$ every 20 minutes at $\mathrm{RT}\left(25^{\circ} \mathrm{C}\right)$. The resultant non-woven mats were air-dried for 48 $h$ at $\mathrm{RT}$, and then peeled off the collector plate manually prior to storage at $25^{\circ} \mathrm{C}$. Fibre morphology $(100$ measurements per scaffold) and scaffold thickness (20 measurements per scaffold) were measured following sputter-coating in gold (4 minutes) on a JEOL SM 1100 scanning electron microscope (accelerating voltages $10-30 \mathrm{kV}$; working distances 15 to $35 \mathrm{~cm}$ ) using the SEM-associated SMiles Image processing software. The static WCAs of the scaffolds (three scaffolds of each batch) were determined from sessile WCA measurements (taken at five different $1 \mathrm{~cm}^{2}$ areas, total scaffold area $=6 \mathrm{~cm}^{2}$ ) taken using distilled water and a CAM 200 sessile drop video capture apparatus from which the average and standard deviation were calculated.

Table 1: Co-electrospinning parameters of different polymer solutions.

\begin{tabular}{l|ccc} 
Polymer solutions & Flow rate & Working distance & Voltage \\
\cline { 1 - 1 } PET-poly $\left(\right.$ PEGMA $\left._{188}\right)$ & $4 \mathrm{~mL} / \mathrm{h}$ & $15 \mathrm{~cm}$ & $19 \mathrm{KV}$ \\
\hline PLGA-poly $\left(\mathrm{PEGMA}_{188}\right)$ & & $15 \mathrm{~cm}$ & $14 \mathrm{KV}$ \\
\hline PET & $0.5 \mathrm{~mL} / \mathrm{h}$ & $15 \mathrm{~cm}$ & $19 \mathrm{KV}$
\end{tabular}

\subsection{Cell culture and maintenance}

Human donor tissue was used with approval by the local ethics research committee and in accordance with the tenets of the declaration of Helsinki, following consent obtained from the donors and/or their relatives. 
Corneoscleral rims were obtained under an MTA from Nottingham University Hospitals Trust and all work performed in accordance with the Human Tissue Authority. Corneoscleral rims arrived fully anonymised, thus age, gender and health status is unknown. Primary human corneal stromal stem cells (hCSSCs) were isolated from corneoscleral rims of three donors using a modification of a previously described method.[19] Cells were cultured in medium-199 supplemented with $20 \%(v / v)$ foetal calf serum (FCS), 100 units $/ \mathrm{mL}$ penicillin, $100 \mu \mathrm{g} / \mathrm{mL}$ streptomycin and 1\% ( $v / v)$ L-glutamine. Green fluorescent protein (GFP)-expressing immortalised human mesenchymal stem cells (ihMSCs; generated in-house), human colon adenocarcinoma cells (Caco2) and RFP-expressing NIH-3T3 fibroblasts were cultured in Dulbecco's modified Eagle's medium supplemented with $10 \%(\mathrm{v} / \mathrm{v}) \mathrm{FCS}, 100 \mathrm{units} / \mathrm{mL}$ penicillin, $100 \mu \mathrm{g} / \mathrm{mL}$ streptomycin and non-essential amino acids. All cells were maintained in a humidified incubator at $37^{\circ} \mathrm{C}$ and $5 \% \mathrm{CO}_{2}$ in air, with medium changes every 2 days.

\subsection{Scaffold sterilization and cell seeding}

Fibrous scaffolds were cut into discs $\left(1 \mathrm{~cm}^{2}\right)$ using a trephine (stainless steel cylinders, $1 \mathrm{~cm}$ diameter) and placed in non-tissue culture treated 6 well plates. Scaffolds were sterilised by UV radiation (254 nm) for 20 minutes on each side then incubated at $37^{\circ} \mathrm{C}$ with $2 \mathrm{~mL}$ of $\mathrm{PBS}$ containing $100 \mu \mathrm{g} / \mathrm{mL}$ of gentamicin/amphotericin (AB/AM), (10 mL of PBS: $100 \mu \mathrm{L}$ of $A B / A M)$ for $24 \mathrm{~h}$. Scaffolds and control surfaces (tissue culture polystyrene; TCPS) were washed with PBS and pre-incubated in culture media for $24 \mathrm{~h}$. Cells were seeded on scaffolds at a density of $1 \times 10^{5} \mathrm{cells} / \mathrm{mL}$ and cultured at $37^{\circ} \mathrm{C}$ in $5 \% \mathrm{CO}_{2}$ in air.

\subsection{Determination of hCSSCs confluence using Cellstain ${ }^{\mathrm{TM}}$ double-staining kit}

Cellstain ${ }^{\mathrm{TM}}$ double staining kit is utilised for simultaneous fluorescence staining of viable (calcein-AM) and dead (propidium iodide) cells and was used as an endpoint assay. The assay solution was prepared by adding $10 \mu \mathrm{L}$ of solution $\mathrm{A}$ (acetoxymethyl ester of calcein) and $5 \mu \mathrm{L}$ of solution B (propidium iodide) to $5 \mathrm{~mL}$ of PBS. Cell-seeded scaffolds and TCPS control surfaces were incubated with $500 \mu \mathrm{L}$ of the assay solution for 15 minutes at $37^{\circ} \mathrm{C}$.

\subsection{Effect of poly(PEGMA $\left.{ }_{188}\right)$ on hCSSCs viability assessed using Alamar $B$ lue ${ }^{\circledR}(A B)$ assay}

Alamar Blue ${ }^{\circledR}$ is a resazurin dye-based assay used as a cell viability indicator. It is non-fluorescent in its oxidised state. However, viable cells have the ability to reduce resazurin to resorufin, a highly fluorescent 
pink dye. Cultured hCSSC viability experiments were conducted over 10 days, with fresh solutions prepared on each day of the assay. On day 0 , the assay was conducted $6 \mathrm{~h}$ after seeding the scaffolds. Scaffolds were transferred to a new 6 non-tissue culture well plate and washed 3 times with warm PBS to remove unattached cells. Aliquots $(1 \mathrm{~mL})$ of $A B$ working solution $(10 \%(\mathrm{v} / \mathrm{v})$ AB stock solution (Invitrogen) in Hanks Balanced Salts Solution (HBSS) without phenol red and serum (Sigma)) was added to each scaffold before incubation at $37^{\circ} \mathrm{C}$ for 90 minutes. After incubation, $200 \mu \mathrm{L}$ aliquots of $\mathrm{AB}$ solution was transferred to 96 well-plates and fluorescence was measured at an excitation/emission wavelength of 560/590 nm. Scaffolds were washed with warm PBS then returned to the same cell culture media and incubated at $37^{\circ} \mathrm{C}$ for day 3 and day 5 readings. Detachment on day 5 (passage 1) was carried out by lowering the temperature to $17^{\circ} \mathrm{C}$ for 7 minutes, with gentle agitation, for both test (thermoresponsive PET or PLGA) and control (PET and PLGA alone) scaffolds. On day 5 post-passaging, new scaffolds were used and re-seeded with $1 \times 10^{5}$ cells $/ \mathrm{mL}$ for $A B$ measurements. Cell detachment was conducted again on the scaffolds (passage 2). Three independent experiments were carried out in triplicate ( $n=9$, three scaffolds from three batches).

\subsection{Prolonged (seven) thermal and enzymatic passages of hCSSCs on thermoresponsive PET- poly(PEGMA $\left.{ }_{188}\right)$ scaffolds and TCPS respectively}

The starting passage number of hCSSCs was 2; cells were grown to $80 \%$ confluency and removed from the TCPS culture surface using TrypLE ${ }^{\mathrm{TM}}$. Cells were pelleted by centrifugation at $200 \mathrm{xg}$ for $5 \mathrm{~min}$, washed and re-suspended in medium. They were then seeded on $1 \mathrm{~cm}^{2}$ co-electrospun thermoresponsive PET scaffolds and 12 well TCPS at a density of $1 \times 10^{5}$ cells $/ \mathrm{mL}$ in a total of $1 \mathrm{~mL}$ of medium, then cultured at $37^{\circ} \mathrm{C}$. The medium was changed the next day. Thermal detachment studies from PET-poly(PEGMA188) scaffolds were carried out after $120 \mathrm{~h}$ of culture (every five days for seven passages, on new scaffolds for each passage; total $=35$ days), where temperature was lowered from $37^{\circ} \mathrm{C}$ to $17^{\circ} \mathrm{C}(\mathrm{RT})$ for 7 minutes (with gentle agitation). Enzymatic detachment of cells from TCPS and scaffolds fabricated from PET-alone was carried out via enzymatic TrypLE ${ }^{\mathrm{TM}}$ treatment for 5 minutes at $37^{\circ} \mathrm{C}$. Cells detached from the scaffolds were gently pipetted to ensure a single cell suspension was obtained. Cells were counted after each passage using a Neubauer haemocytometer chamber. Population doublings (PDs) at each passage were calculated using Equation (1):

PDs $=\left[\log _{10}(\right.$ total cell counts/cells seeded $\left.) / \log _{10} 2\right]$ 
where the initial cell number seeded was $1 \times 10^{5} \mathrm{cells} / \mathrm{mL}$. Proliferation rates were calculated as cumulative population doublings (CPDs) as a function of time ( $n=9$ total, three scaffolds from three different batches).

\subsection{RNA extraction, cDNA synthesis and quantitative reverse transcription-polymerase chain reaction (RT-qPCR)}

Total RNA was extracted from hCSSCs cultured on scaffolds and TCPS ( $n=3$ each) using RNeasy Mini Kit (Qiagen). Samples from non-thermoresponsive scaffolds (PET and PLGA) were chopped into small pieces using a scalpel before treatment. Total RNA for each sample (300 ng) was reverse-transcribed into singlestranded cDNA using Superscript III reverse transcriptase (Life Technologies, UK) with random hexamer primers, according to the manufacturer's protocol. PCR reactions were conducted using the prepared cDNA $(2 \mu \mathrm{L})$ with inventoried Taqman assays (Applied Biosystems, Life Technologies) to detect (GAPDH; Hs99999905_m1), CD34 (Hs00990732_m1), THY1 (CD90; Hs00174816_m1), ACTA2 (Hs00426835_g1) and ALDH3A1 (Hs00964880_m1). Amplification was performed on an Mx3005P multicolour 96-well PCR system (Stratagene, Agilent Technologies, UK). Mixtures were cycled as follows: $50^{\circ} \mathrm{C}$ for 2 minutes for one cycle (initial denaturation), one cycle at $95^{\circ} \mathrm{C}$ for 10 minutes (denaturing), then 50 cycles at $95^{\circ} \mathrm{C}$ for 15 seconds (annealing) and $60^{\circ} \mathrm{C}$ for 1 minute (extension). RT-qPCR reactions were analysed using Real Time PCR Miner algorithm[20] which calculates efficiency and threshold cycle. All experimental values were normalised to readings of the endogenous reference gene, glyceraldehyde 3-phosphate dehydrogenase (GAPDH). GAPDH as an endogenous reference gene for the hCSSC has been confirmed previously by our group. [25, 27]

\subsection{Immunocytochemical staining}

Cells on PET-4\% poly(PEGMA188) and TCPS surfaces were fixed using $4 \%(w / v)$ paraformaldehyde for 10 minutes and permeabilised in $100 \%(\mathrm{~V} / \mathrm{v})$ methanol for 10 minutes. Blocking was performed for 1 hour at RT in PBS with 1\% $(v / v)$ bovine serum albumin (BSA), $0.3 \mathrm{M}$ glycine and 3\% $(v / v)$ donkey serum. Samples were incubated with mouse monoclonal primary antibodies at $4^{\circ} \mathrm{C}$ overnight (CD34, 1:200 and $\alpha$-SMA, 1:200). After washing, samples were incubated with secondary Alexa Fluor 488 donkey anti-mouse IgG (1:300) for $1 \mathrm{~h}$. Samples were counterstained with $1 \mathrm{mg} / \mathrm{mL}$ propidium iodide. Samples were mounted in fluorescence mounting medium (Dako, UK) before imaging. Images were obtained using a Nikon Eclipse TS 100 microscope and a DS-L3 Nikon capture unit. 


\subsection{Statistical analysis}

Statistical analyses were performed using GraphPad Prism 6 software (San Diego, CA) following confirmation of normal data distribution. Two groups were compared using unpaired Student's $t$-test and multiple groups were compared using one- or two-way ANOVA with post-hoc Dunnett's test. A value of $p \leq$ 0.05 was considered significant.

\section{Results}

Beadless fibres with an average diameter of $\sim 840 \pm 170 \mathrm{~nm}$ and $3 \pm 0.8 \mu \mathrm{m}$ were prepared from $30 \%(W / v)$ PET and $18.5 \%(w / v)$ PLGA, respectively. This was achieved by altering various electrospinning parameters such as polymer solution concentration, voltage and flow rate (Table 1). Surface wettability of the scaffolds was determined by WCA measurements at $20^{\circ} \mathrm{C}$. The average wettability $\left(\theta_{\mathrm{w}}\right)$ of the PET and PLGA scaffolds alone was low (105 $\pm 5^{\circ}$ and $131 \pm 10^{\circ}$, respectively), indicating a hydrophobic surface (Figure 2). Next, a dodecane-thioether terminated poly(poly(ethylene glycol)methacrylate) (poly(PEGMA 188$)$ ) polymer was synthesized by free radical polymerization following a published method.[18] ${ }^{1} \mathrm{H}$ NMR and ${ }^{13} \mathrm{C}$ NMR were conducted to confirm the successful preparation and purification of poly(PEGMA 188 ) (Figure S1a and b). Gel permeation chromatography (GPC) confirmed the molar mass of the polymer $\left(M_{\mathrm{w}}(\mathrm{GPC})=51 \mathrm{kDa}\right.$, $\Xi=1.8$, Figure S1d). The synthesised polymer solution transparency changed from clear to turbid at $26^{\circ} \mathrm{C}$. Spectrophotometry was also used to determine the cloud point temperature of poly(PEGMA 188$)$, which was found to be between $22.5^{\circ} \mathrm{C}$ and $25.2^{\circ} \mathrm{C}$ (Figure S1C).

The second step involved the incorporation of $4 \%(w / v)$ poly $\left(\mathrm{PEGMA}_{188}\right)$ with either the PET or PLGA polymer solutions for co-electrospinning (blend-electrospinning) to form the $3 \mathrm{D}$ thermoresponsive coelectrospun scaffolds. Scanning electron microscopy (SEM) analysis of the resultant scaffolds (Figure 2) showed that the addition of the thermoresponsive polymer did not affect fibre morphology or bead formation. The average fibre diameters of the co-electrospun scaffolds of PET-poly(PEGMA 188 ) and PLGApoly $\left(\right.$ PEGMA $\left._{188}\right)$ did increase to $870 \pm 195 \mathrm{~nm}$ and $3.8 \pm 0.45 \mu \mathrm{m}$ respectively, compared to PET or PLGA scaffolds alone. The thickness of all scaffolds was on average $43.4 \pm 2.45 \mu \mathrm{m}$ (as measured by SEM). The density of the fibres were consistent between scaffolds within a batch and from one batch to another because all of the electrospun scaffolds were prepared under the same electrospinning conditions. 
WCA measurements were conducted on co-electrospun fibres at $37^{\circ} \mathrm{C}, 20^{\circ} \mathrm{C}$ and $17^{\circ} \mathrm{C}$, as shown in Figure 2 and Table 2, with WCAs at $20^{\circ} \mathrm{C}$ displaying a change from the hydrophobic nature of the PET and PLGA fibres (105 $\pm 5^{\circ}$ and $131 \pm 10^{\circ}$, respectively) to a more hydrophilic surface $\left(74 \pm 12^{\circ}\right.$ and $75 \pm 13^{\circ}$, respectively). ${ }^{1} \mathrm{H}-\mathrm{NMR}$ analysis of the dissolved co-electrospun fibres was conducted to investigate the elemental composition of these surfaces and confirm the presence of poly (PEGMA 188$)$ in the fibres. Peaks attributable to poly(PEGMA $\left.{ }_{188}\right)$ were observed between 3.4-4.3 ppm, as shown in Figures S2 and S3 for coelectrospun PET-poly(PEGMA 188$)$ and PLGA-poly(PEGMA 188$)$, respectively.

Table 2: WCA analysis on the different polymers under investigation, carried out at various temperatures ( $n=3$ batches of scaffolds in triplicate).

\begin{tabular}{l|c|c|c} 
Scaffold Composition & At $\mathbf{3} 7^{\circ} \mathbf{C}$ & At $\mathbf{2 0}{ }^{\circ} \mathbf{C}$ & At $\mathbf{1 7}{ }^{\circ} \mathbf{C}$ \\
\hline $30 \%$ PET: 0\% Poly(PEGMA 188$)$ & $110 \pm 4^{\circ}$ & $105 \pm 5^{\circ}$ & $107 \pm 3^{\circ}$ \\
\hline $30 \%$ PET: $4 \%$ Poly(PEGMA 188$)$ & $112 \pm 10^{\circ}$ & $74 \pm 12^{\circ}$ & $62 \pm 7^{\circ}$ \\
\hline $18.5 \%$ PLGA: 0\% Poly(PEGMA 188$)$ & $132.5 \pm 9^{\circ}$ & $131 \pm 10^{\circ}$ & $129 \pm 10^{\circ}$ \\
\hline $18.5 \%$ PLGA: $\% \%$ Poly(PEGMA 188$)$ & $129 \pm 3^{\circ}$ & $75 \pm 13^{\circ}$ & $72 \pm 14^{\circ}$
\end{tabular}

Following synthesis and characterisation, the ability to culture cells within the 3D thermoresponsive electrospun scaffolds was evaluated. The application of this novel thermoresponsive system for the culture of primary human CSSCs was assessed; these cells were chosen as an exemplar therapeutically relevant cell type. These experiments were carried out on both the PET and PLGA scaffold systems and findings were comparable. For simplicity we describe the results from the PET scaffolds here and present the data from the PLGA scaffolds in supplementary information (Figure S7). Staining of hCSSCs cultured on PET and PET-poly(PEGMA 188 ) scaffolds and tissue culture polystyrene (TCPS) with Cellstain ${ }^{\mathrm{TM}}$ double-staining kit was carried out. The cells required almost five days to become $70 \%$ confluent, then were detached at $17^{\circ} \mathrm{C}(\mathrm{RT})$ without the use of trypsin (Figure 3a). Notably, hCSSCs remained viable on the thermoresponsive polymer poly $\left(\mathrm{PEGMA}_{188}\right)$ scaffolds with no cytotoxicity evident, and proliferated in a similar manner to those cultured on TCPS surfaces. There was a high degree of cell-cell contact on PET-containing scaffolds (Figure 3a) and the dendritic morphology of hCSSCs was maintained.

The viability of hCSSCs was assessed over five days in culture. A significant increase in fluorescence intensity ( $p \leq 0.0001$ ) was observed from day 0 to 3 and from days 3 to 5 at $37^{\circ} \mathrm{C}$ on all electrospun scaffolds 
and on TCPS (Figure 3b). These results indicate that hCSSCs were viable over five days in culture and confirm the biocompatibility of the scaffolds, as they supported cell proliferation in a similar manner to TCPS. The AB assay was also conducted to determine the thermoresponsive behaviour of co-electrospun scaffolds by detecting change in fluorescence intensity while changing the culture temperature periodically over a total of 10 days in culture ( 2 passages every 5 days using new scaffolds at each passage; Figure $3 \mathbf{c}$ ). A significant decrease in fluorescence intensity was observed following a change of culture temperature of the PET-4\% poly(PEGMA) scaffolds, which indicated that detachment of cells from the culture surface had occurred. Post-passage 1, there was a significant decrease in the fluorescence intensity $(p \leq 0.0001)$ generated by the cells when the poly(PEGMA188)-containing scaffolds were incubated at $17^{\circ} \mathrm{C}$, while the fluorescence intensity generated by cells on the PET scaffolds alone and TCPS at day 5 (pre-passage 1 before detachment) and post-passage 1 were not significantly different $(p>0.05)$. On day 5 post-passage 1 , a set of new scaffolds were used, reseeded with $1 \times 10^{5} \mathrm{cells} / \mathrm{mL}$ and cultured for 5 days at $37^{\circ} \mathrm{C}$ for the Alamar Blue ${ }^{\circledR}$ measurements. Cell detachment was conducted again on the scaffolds (Passage 2). After five days of culture of the re-seeded scaffolds, detachment occurred after 7 minutes at $17^{\circ} \mathrm{C}$ with gentle agitation / passage 1 and passage 2 (mean \pm SD; $n=9$ ). The fluorescence intensity on PET scaffolds and TCPS at day 5 and passage 1 , or at day 5 post-passage and passage 2 were not significantly different as the cells remained adherent at the reduced temperature.

Cells harvested from thermoresponsive PET-poly(PEGMA 188$)$ scaffolds and TCPS, via reducing the culture temperature or trypsin digestion, respectively, were counted using a haemocytometer at each passage (total of nine passages), as shown in Figure 4a. Statistically significant differences in cell numbers between the thermoresponsive scaffolds and TCPS were observed at passages 1 and 3 only suggesting a lag phase as cells adjusted to the 3D environment. At passages $2,4,5,6,7,8$ and 9 there was no significant difference between cell numbers harvested from TCPS when compared with thermal detachment from the 3D scaffolds. Population doublings (PDs) were also calculated at each passage Figure $\mathbf{4 b}$. The average PD interval on TCPS was $2.8 \pm 0.75$ days and on PET-poly $\left(P E_{\text {GMA }}{ }_{188}\right)$ was $3.6 \pm 1.2$ days. No significant difference in CPDs was observed up to 20 days in culture, but there were significant differences in the CPDs observed on days 25 and $30(p \leq 0.01)$, day $35(p \leq 0.001)$ and days 40 and $45(p \leq 0.0001)$ in culture between scaffolds and TCPS. This indicates that the growth and proliferation of hCSSCs on the thermoresponsive co-electrospun scaffolds was similar to that on 2D TCPS but slowed during the latter phases of the culture period. 
RT-qPCR was carried out for CD34, ALDH3A1, TYH1(CD90) and ACTA2 to evaluate the effect of the 3D environment provided by the thermoresponsive scaffold on cell phenotype in comparison with those cultured on 2D surfaces (Figure 4c). There were no significant differences in mRNA levels of CD34, ALDH3A1, THY1 and ACTA2 expressed by cells cultured on either of the thermoresponsive scaffolds when compared with the non-thermoresponsive electrospun scaffolds (PET alone). This indicates that the thermoresponsive poly $\left(\right.$ PEGMA $\left._{188}\right)$ did not elicit a negative effect on the cultured hCSSC phenotype. Moreover, data showed that mRNA levels of $C D 34$ and $A L D H 3 A 1$ were significantly higher $(p \leq 0.05)$ when cells were cultured on all 3D electrospun scaffolds relative to those cultured on 2D TCPS, with the complete absence of CD34 expression in cells cultured on TCPS from passage 3. For ACTA2, mRNA levels were significantly lower $(p \leq 0.05)$ when cells were cultured on all electrospun scaffolds relative to those cultured on TCPS. For THY1 (CD90), there were no significant differences in mRNA levels between cells cultured on all electrospun scaffolds relative to those cultured on TCPS.

Prolonged cell culture and serial passaging experiments were conducted to evaluate the impact of extended culture of hCSSCs on the 3D thermoresponsive scaffolds and to assess effects on gene expression of corneal stromal stem cells in comparison with 2D TCPS. hCSSCs were seeded at passage 2 on TCPS (2D) and on PET-poly(PEGMA 188 ) scaffolds (3D) and cultured for a total of 35 days. During this period, cells were passaged seven times using either thermal (3D scaffolds) or enzymatic (2D TCPS) methods. RT-qPCR was performed on hCSSCs at passages 3,6 and 9 to determine relative differences in mRNA levels of CD34, ALDH3A1, THY1 and ACTA2. Cells from three different human donors were used for this study, with cells from each donor cultured on both 3D and 2D substrates (i.e. matched donors).

Significant differences in mRNA levels were seen in cells cultured on 2D surfaces when compared with those cultured on 3D scaffolds (Figure 5a). CD34 and ALDH3A mRNA expression levels were significantly higher ( $p \leq 0.05$ and $p \leq 0.01$ respectively) in cells cultured on 3D thermoresponsive scaffolds than those cultured on 2D TCPS for all passages (3, 6 and 9) and for all three donors. CD34 gene expression was detected in cells from two donors only at passage 6 and 9 when cultured on 2D surfaces, in comparison with 3D scaffolds where CD34 gene expression was maintained in all donors. For THY1 (CD90), there were no significant differences in mRNA levels between cells cultured on 3D scaffolds and 2D TCPS over all passages. For ACTA2, there was a significant decrease in gene expression when cells were cultured on 3D scaffolds when 
compared with 2D at passages 6 and 9 ( $p \leq 0.01$ and $p \leq 0.001$ respectively), with no significant difference observed at passage 3 .

Immunocytochemistry was conducted in parallel to RT-qPCR at passages 3,6 and 9 for cells cultured on TCPS and PET-poly(PEGMA 188$)$ to identify the expression of key markers associated with quiescent keratocytes (CD34) and activated myofibroblasts ( $\alpha$-SMA). Results of RT-qPCR studies were confirmed by immunocytochemistry (Figure 5b). CD34-positive staining was observed in all cells at all passages when cultured on 3D thermoresponsive scaffolds, while this expression was lost after passage 3 when cultured on 2D TCPS. The myofibroblast protein, $\alpha$-SMA, is visible in individual cells cultured on TCPS at all passages but not in cells cultured on 3D scaffolds.

Data for prolonged culture of hCSSCs on 2D TCPS and 3D scaffolds are also presented relative to TCPS at passage 3 to explore any change in phenotypic marker expression with increasing passage number (Figure 6). $C D 34$ and $A L D H 3 A 1$ mRNA levels were significantly lower ( $p \leq 0.05$ and $p \leq 0.0001$, respectively) at passage 6 and 9 (compared with levels expressed at passage 3). No significant difference was observed between levels expressed at passage 3 in comparison to passage 6 and 9 in cells cultured on both 2D TCPS and 3D electrospun scaffolds. However, CD34 expression was completely lost in one of the donor hCSSCs cultured on TCPS after passage 3. For THY1 (CD90), mRNA levels were significantly higher $(p \leq 0.0001)$ at passage 9 than earlier cell passages. However, THY1 is expressed by cells throughout this in vitro study regardless of the culture environment, and expression was not lost during their 2D culture. For ACTA2, mRNA levels were significantly decreased $(p \leq 0.05)$ from passage 3 to passage 6 and from passage 6 to passage 9 in cells cultured on both 2D TCPS and 3D scaffolds.

These scaffolds were then tested with other cell types to illustrate that this thermoresponsive system can be used for the expansion of various mammalian cell types (Figure $\mathbf{S 4} \mathbf{- S 6 )}$ ). The attachment, spreading and proliferation of $\mathrm{NIH}-3 \mathrm{~T} 3$ fibroblasts was similar when compared to those cultured on non-thermoresponsive scaffolds. Moreover, detachment of the cultured cells from thermoresponsive scaffolds was achieved after reducing the culture temperature to $17^{\circ} \mathrm{C}(\mathrm{RT})$ over 7 minutes (with gentle agitation; Figure S4). Prolonged culture and passaging of $\mathrm{NIH}-3 \mathrm{~T} 3$ cells (over 10 passage cycles) was conducted illustrating similar growth curve profiles on the 3D scaffolds compared with TCPS (Figure S4c and d). Further experiments with 
human colon adenocarcinoma cells (Caco2; Figure S5) and human mesenchymal stem cells (Figure S6) showed attachment, proliferation and detachment over ten days in culture for both cell types. Both cell types were viable over the time-course of the experiment, as confirmed by the Alamar blue (AB) assay.

\section{Discussion}

The aim of this study was to develop a 3D culture system that can be used for the extended maintenance and enzyme-free passaging of anchorage-dependent mammalian cells at physiological conditions. The first step in this study involved the electrospinning and characterization of PET and PLGA fibre-based scaffolds chosen as the 3D environment. Beadless fibres were prepared from 30\% (w/v) PET and 18.5\% ( $w / v)$ PLGA. poly(PEGMA $\left.{ }_{188}\right)$ was chosen as the thermoresponsive polymer due to its history of use in drug delivery and known biocompatibility. In addition, process of cell attachment/detachment is unhindered from PEG-based substrates by irreversible dehydration. The synthesised polymer showed thermoresponsive properties, as shown by the cloud point temperature (as a proxy for the LCST) of poly (PEGMA ${ }_{188}$ ), which was found to be between $22.5^{\circ} \mathrm{C}$ and $25.2^{\circ} \mathrm{C}$. Surface wettability is an important factor for cell adhesion and subsequent recovery from the culture surface.[21, 22] WCA measurements showed a change from the hydrophobic nature of the PET and PLGA fibres to a more hydrophilic surface.

Following synthesis and characterisation, the ability to culture cells within the 3D thermoresponsive electrospun scaffolds was evaluated. Poly(PEGMA 188$)$-containing scaffolds exhibit a porous structure (Figure 2), which provides a fibrous tissue-like extracellular environment for cells to interact. These scaffolds provided a 3D culture system in comparison with TCPS, which is planar and non-porous. The detachment of the cells from the scaffold is thought to be controlled via the surface hydration of the temperatureresponsive electrospun scaffolds as the temperature is decreased from $37^{\circ} \mathrm{C}$ to $17^{\circ} \mathrm{C}$. This surface hydration can be explained in relation to the thermo-responsive polymer chain extension and contraction that accompanies the change in temperature. Increasing the culture temperature above the polymer's LCST, collapses the poly $\left(\mathrm{PEGMA}_{188}\right)$ chains, generating a strong hydrophobic surface that is supportive of cell attachment. Below its LCST, poly $\left(\mathrm{PEGMA}_{188}\right)$ is water-soluble with an amphiphilic character, due to its hydrophobic methacrylate backbone and hydrophilic pendant PEG side chains. This leads to the formation of a hydration layer between the scaffold fibre surface and the cultured cells, which results in cell detachment from the thermo-responsive scaffolds. The hydrophilicity of the same surface can be reversed by increasing 
the temperature above the LCST, which results in dehydration of the poly $\left(\mathrm{PEGMA}_{188}\right)$ chains, leading to their collapse. Cells adhered to the thermoresponsive substrates under investigation at $37^{\circ} \mathrm{C}$, since these thermoresponsive cell culture systems are hydrophobic at this temperature (> LCST). Detachment of cells was induced at ambient temperatures $\left(17^{\circ} \mathrm{C}\right)$ as the substrate becomes hydrophilic, inhibiting cell adhesion (Figure 1).

Clinically relevant human corneal stromal stem cells (hCSSCs) have been investigated for use in stromal wound healing and regeneration.[23-25] Their phenotype has been well characterised and it is known that they undergo (myo)fibroblastic differentiation when expanded on 2D surfaces in serum-containing media.[27] Moreover, difficulties have been reported in extracting these cells from PLGA electrospun scaffolds;[17] all these aspects led to us investigating the application of this novel thermoresponsive system for the culture of hCSSCs. Cells were cultured in M199 medium supplemented with $20 \%(\mathrm{v} / \mathrm{v})$ serum, which has been proven to support hCSSC proliferation.[27] Staining of hCSSCs cultured on PET, PETpoly(PEGMA 188$)$, PLGA, PLGA-poly(PEGMA $\left.{ }_{188}\right)$ scaffolds and tissue culture polystyrene (TCPS) with Cellstain $^{\mathrm{TM}}$ double-staining kit was carried out to confirm cell confluency on the scaffolds. Notably, hCSSCs remained viable on the thermoresponsive polymer poly(PEGMA $\left.{ }_{188}\right)$ with no cytotoxicity evident, and proliferated in a similar manner to those cultured on TCPS surfaces.

Further work demonstrated that hCSSCs were viable over five days in culture and confirmed the biocompatibility of the scaffolds, as they supported cell proliferation in a similar manner to TCPS. The AB assay also confirmed the thermoresponsive behaviour of co-electrospun scaffolds, depicted by the change in fluorescence intensity while changing the culture temperature periodically over a total of 10 days in culture. Statistically significant differences in cell numbers between thermoresponsive scaffolds and TCPS were observed at passages 1 and 3 only, which suggests the incomplete recovery of attached hCSSCs cells from the scaffolds. However, there was no significant difference between cell numbers harvested from TCPS when compared with thermal detachment from the 3D scaffolds at passages $2,4,5,6,7,8$ and 9 . No significant difference in CPDs was observed up to 20 days in culture, but there were significant differences in the CPDs observed afterwards between scaffolds and TCPS. This indicates that the growth and proliferation of hCSSCs on the thermoresponsive co-electrospun scaffolds was similar to that on 2D TCPS but slowed down during the latter phases of the culture period. This is indicative of the maintenance of a more quiescent phenotype of these cells when cultured in 3D associated with a slower proliferation rate. 
The maintenance of the keratocyte phenotype of hCSSCs is an important step towards their use in cellbased clinical applications and research.[28, 29] Therefore, RT-qPCR was carried out for CD34 and $A L D H 3 A 1$ as quiescent keratocyte markers,[19, 30-32] $T Y H 1(C D 90)$ as a MSC/fibroblast marker[33] and ACTA2 as a myofibroblast marker[34] to evaluate the effect of the $3 \mathrm{D}$ environment provided by the thermoresponsive scaffold on cell phenotype in comparison with culture on 2D surfaces. There were no significant differences in mRNA levels of $C D 34, A L D H 3 A 1, T H Y 1$ and ACTA2 expressed by cells cultured on either of the thermoresponsive scaffolds (PET or PLGA) when compared with the non-thermoresponsive electrospun scaffolds. This indicates that the thermoresponsive poly $\left(\mathrm{PEGMA}_{188}\right)$ itself did not elicit a negative effect on the cultured hCSSC phenotype. The results obtained suggest that cell culture on the 3D scaffolds promoted the quiescent keratocyte phenotype, with increased expression of $C D 34$ and $A L D H$ markers, and decreased expression of ACTA2 markers in comparison to conventional 2D culture. Moreover, cells were cultured in $20 \%(\mathrm{~V} / \mathrm{V})$ serum, which supports a highly proliferative phenotype and loss of quiescence as observed in 2D; in contrast, the quiescent phenotype is maintained when cultured in 3D (even in the presence of $20 \%(\mathrm{v} / \mathrm{v})$ serum).

Prolonged cell culture and serial passaging experiments were conducted to evaluate the impact of extended culture of hCSSCs on the 3D thermoresponsive PET-poly(PEGMA 188$)$ scaffolds and to assess effects on gene expression of hCSSC in comparison with 2D TCPS. RT-qPCR was performed on hCSSCs at passages 3,6 and 9 to determine relative differences in mRNA levels of CD34, ALDH3A1, THY1 and ACTA2. Significant differences in mRNA levels were seen in cells cultured on 2D surfaces when compared with those cultured on 3D scaffolds. $C D 34$ and $A L D H 3 A$ mRNA expression levels were significantly higher $(p \leq 0.05$ and $p \leq 0.01$ respectively) in cells cultured on $3 \mathrm{D}$ thermoresponsive scaffolds than those cultured on $2 \mathrm{D}$ TCPS for all passages (3, 6 and 9) and for all three donors. CD34 gene expression was detected in cells from two donors only at passage 6 and 9 when cultured on 2D surfaces, in comparison with 3D scaffolds where CD34 gene expression was maintained in all donors. These results indicate that the quiescent keratocyte phenotype was maintained on PET-poly(PEGMA $\left.{ }_{188}\right)$ scaffolds over 35 days of cell culture. For THY1 (CD90), there were no significant differences in mRNA levels between cells cultured on 3D scaffolds and 2D TCPS over all passages. For ACTA2, there was a significant decrease in gene expression when cells were cultured on 3D scaffolds when compared with 2D at passages 6 and $9(p \leq 0.01$ and $p \leq 0.001$ respectively), with no significant difference observed at passage 3. 
Immunocytochemistry was conducted in parallel to RT-qPCR at passages 3,6 and 9 for cells cultured on TCPS and PET-poly(PEGMA $\left.{ }_{188}\right)$ to identify the expression of key markers associated with quiescent keratocytes (CD34) [30] and activated myofibroblasts (a-SMA).[28, 35] Results of RT-qPCR studies were confirmed by immunocytochemistry. CD34-positive staining was observed in all cells at all passages when cultured on 3D scaffolds, while this expression was lost after passage 3 when cultured on 2D surfaces. The myofibroblast protein, $\alpha$-SMA, is visible in individual cells cultured on TCPS at all passages, but not in cells cultured on 3D scaffolds. These results suggest that the 3D thermoresponsive electrospun scaffolds may be advantageous for the expansion of clinically-relevant cell types without loss of a therapeutically desirable phenotype.

Data for prolonged culture of hCSSCs on 2D TCPS and 3D scaffolds were also presented relative to TCPS at passage 3 to explore any change in phenotypic marker expression with increasing passage number. No significant difference was observed between levels expressed at passage 3 in comparison to passages 6 and 9 in cells cultured on both 2D TCPS and 3D scaffolds, suggesting that the overall population of cells expressing these markers reduces over time. THY1 was expressed by cells throughout this in vitro study regardless of the culture environment, and expression was not lost during their $2 \mathrm{D}$ culture as has been observed previously.[25] Downregulation of the expression of ACTA2 over different passages is a promising finding, as ACTA2 gene expression is related to the undesirable activated corneal myofibroblast phenotype, and this phenomenon has not been previously reported.

These scaffolds were then tested with other cell types to illustrate that this thermoresponsive system can be used for the expansion of various mammalian cells. These scaffolds were able to support the attachment, spreading and proliferation of NIH-3T3 fibroblasts in a similar manner to those cultured on nonthermoresponsive scaffolds. Assessment of integrin and FAK expression (involved in cell adhesion) will be explored in future studies. In addition, the fibrous structure and pore size of the scaffolds can be changed by altering the electrospinning parameters to allow further penetration of the cells into the fibrous structure. Moreover, detachment of the cultured cells from thermoresponsive scaffolds was achieved after reducing the culture temperature to $17^{\circ} \mathrm{C}$ over 7 minutes. Results also demonstrated attachment, proliferation and detachment over ten days in culture for human mesenchymal stem cells (maintenance of the differentiation potential will be a focus in future work) and human colonic adenocarcinoma cells. These data illustrate the 
versatile nature of this culture system as any mammalian cell type can be cultured and expanded on these materials. Further optimisation of the system is a focus of our future work to maximise the fibrous nature of the scaffold in terms of its influence on cell phenotype and scope to provide a high surface area for cell culture.

\section{Conclusions}

This study has demonstrated that blend electrospinning (co-electrospinning) of poly(PEGMA 188 ) with PET or PLGA provides fibres with thermoresponsive surfaces. This method is versatile due to the thermoresponsive fibres being generated from polymer blends rather than being reliant on covalent linkage of the thermoresponsive component to the bulk polymer. This means that thermoresponsive fibres can be generated from any polymer amenable to electrospinning. These scaffolds were able to support mammalian cell adhesion and proliferation at $37^{\circ} \mathrm{C}$ in a similar manner to those cultured on control surfaces. Moreover, this study demonstrated that the thermoresponsive 3D scaffolds supported the culture and detachment of the clinically relevant primary human cells (hCSSC), and other mammalian cell types, without the use of proteolytic enzymes, while promoting a desirable quiescent keratocyte phenotype over multiple passages. This culture system therefore presents a flexible platform for biomedical applications and in particular for expansion of cell types destined for clinical applications.

\section{Acknowledgements}

This work was supported by the King Abdullah Scholarship for Higher Education fund awarded to AMA. CA thanks the Engineering and Physical Sciences Research Council (EPSRC: Grants EP/H005625/1, EP/J021180/1, EP/I01375X/1 and EP/N03371X/1) and the Royal Society for a Wolfson Research Merit Award (WM150086). This work was further supported by the EPSRC Impact Acceleration Account fund awarded to the University of Nottingham (EP/K503800/1). We also thank Jamie Patient (PET NMR), Tom Booth, Paul Cooling, Esme Ireson, Teresa Marshall and Christine Grainger-Boultby for technical support.

Electronic Supplementary Information (ESI) available: All raw data created during this research are openly available from the corresponding author (felicity.rose@nottingham.ac.uk) and at the University of Nottingham Research Data Management Repository (https://rdmc.nottingham.ac.uk/) and all analysed data supporting this study are provided as ESI† accompanying this paper. 


\section{Disclosure statement}

The authors declare no competing interests in this work.

\section{References}

[1] K. Nakajima, S. Honda, Y. Nakamura, F. Lopez-Redondo, S. Kohsaka, M. Yamato, A. Kikuchi, T. Okano, Intact microglia are cultured and non-invasively harvested without pathological activation using a novel cultured cell recovery method, Biomaterials 22(11) (2001) 1213-23.

[2] S. Dey, B. Kellam, M.R. Alexander, C. Alexander, F.R. Rose, Enzyme-passage free culture of mouse embryonic stem cells on thermo-responsive polymer surfaces, Journal of Materials Chemistry 21(19) (2011) 6883-6890.

[3] C. Hanson, G. Caisander, Human embryonic stem cells and chromosome stability, Apmis 113(11-12) (2005) $751-755$.

[4] N. Yamada, T. Okano, H. Sakai, F. Karikusa, Y. Sawasaki, Y. Sakurai, Thermo-responsive polymeric surfaces; control of attachment and detachment of cultured cells, Die Makromolekulare Chemie, Rapid Communications 11(11) (1990) $571-576$.

[5] C. de las Heras Alarcón, S. Pennadam, C. Alexander, Stimuli responsive polymers for biomedical applications, Chemical Society Reviews 34(3) (2005) 276-285.

[6] Z.L. Tang, Y. Akiyama, T. Okano, Temperature-Responsive Polymer Modified Surface for Cell Sheet Engineering, PolymersBasel 4(3) (2012) 1478-1498.

[7] Y. Miyahara, N. Nagaya, M. Kataoka, B. Yanagawa, K. Tanaka, H. Hao, K. Ishino, H. Ishida, T. Shimizu, K. Kangawa, Monolayered mesenchymal stem cells repair scarred myocardium after myocardial infarction, Nature medicine 12(4) (2006) 459-465.

[8] N. Jaiswal, S.E. Haynesworth, A.I. Caplan, S.P. Bruder, Osteogenic differentiation of purified, culture-expanded human mesenchymal stem cells in vitro, J Cell Biochem 64(2) (1997) 295-312.

[9] H.W. Schnaper, D.S. Grant, W.G. Stetler-Stevenson, R. Fridman, G. D'Orazi, A.N. Murphy, R.E. Bird, M. Hoythya, T.R. Fuerst, D.L. French, et al., Type IV collagenase(s) and TIMPs modulate endothelial cell morphogenesis in vitro, J Cell Physiol 156(2) (1993) 235-46.

[10] C.A. Redi, 3D cell culture - Methods and protocols, 2011.

[11] Z.-M. Huang, Y.-Z. Zhang, M. Kotaki, S. Ramakrishna, A review on polymer nanofibers by electrospinning and their applications in nanocomposites, Composites science and technology 63(15) (2003) 2223-2253.

[12] E. Wischerhoff, K. Uhlig, A. Lankenau, H.G. Börner, A. Laschewsky, C. Duschl, J.F. Lutz, Controlled Cell Adhesion on PEGBased Switchable Surfaces, Angewandte Chemie International Edition 47(30) (2008) 5666-5668.

[13] J.-F. Lutz, Ö. Akdemir, A. Hoth, Point by point comparison of two thermosensitive polymers exhibiting a similar LCST: is the age of poly (NIPAM) over?, Journal of the American Chemical Society 128(40) (2006) 13046-13047.

[14] J.L. Funderburgh, M.M. Mann, M.L. Funderburgh, Keratocyte phenotype mediates proteoglycan structure: a role for fibroblasts in corneal fibrosis, The Journal of biological chemistry 278(46) (2003) 45629-37.

[15] J.A. West-Mays, D.J. Dwivedi, The keratocyte: corneal stromal cell with variable repair phenotypes, The international journal of biochemistry \& cell biology 38(10) (2006) 1625-1631.

[16] R.E. Thompson, L.C. Boraas, M. Sowder, M.K. Bechtel, E.J. Orwin, Three-dimensional cell culture environment promotes partial recovery of the native corneal keratocyte phenotype from a subcultured population. Tissue Engineering Part A 19(1314) (2013) 1564-1572.

[17] S. Dunphy, Development and evaluation of a bilayered electrospun scaffold for the anterior cornea, School of Pharmacy, University of Nottingham, 2015.

[18] R.C. Al Ghanami, B. Saunders, C. Bosquillon, K. Shakesheff, C. Alexander, Responsive particulate dispersions for reversible building and deconstruction of 3D cell environments, Soft Matter 6(20) (2010) 5037-5044.

[19] K. Hashmani, M.J. Branch, L.E. Sidney, P.S. Dhillon, M. Verma, O.D. Mclntosh, A. Hopkinson, H.S. Dua, Characterization of corneal stromal stem cells with the potential for epithelial transdifferentiation, Stem Cell Res Ther 4(75) (2013) 927-936.

[20] S. Zhao, R.D. Fernald, Comprehensive algorithm for quantitative real-time polymerase chain reaction, Journal of computational biology 12(8) (2005) 1047-1064.

[21] K. Webb, V. Hlady, P.A. Tresco, Relative importance of surface wettability and charged functional groups on NIH 3T3 fibroblast attachment, spreading, and cytoskeletal organization, Journal of biomedical materials research 41(3) (1998) 422.

[22] Y. Arima, H. Iwata, Effect of wettability and surface functional groups on protein adsorption and cell adhesion using welldefined mixed self-assembled monolayers, Biomaterials 28(20) (2007) 3074-3082.

[23] D.G. Said, M. Nubile, T. Alomar, A. Hopkinson, T. Gray, J. Lowe, H.S. Dua, Histologic features of transplanted amniotic membrane: implications for corneal wound healing, Ophthalmology 116(7) (2009) 1287-95. 
[24] M. Nubile, H.S. Dua, M. Lanzini, M. Ciancaglini, R. Calienno, D.G. Said, A. Pocobelli, R. Mastropasqua, P. Carpineto, In vivo analysis of stromal integration of multilayer amniotic membrane transplantation in corneal ulcers, American journal of ophthalmology 151(5) (2011) 809-822 e1.

[25] L.E. Sidney, O.D. McIntosh, A. Hopkinson, Phenotypic Change and Induction of Cytokeratin Expression During In Vitro Culture of Corneal Stromal Cells Cytokeratin Expression by Corneal Stromal Cells, Investigative ophthalmology \& visual science 56(12) (2015) 7225-7235.

[26] N. Pinnamaneni, J.L. Funderburgh, Concise review: Stem cells in the corneal stroma, Stem cells 30(6) (2012) $1059-1063$.

[27] L.E. Sidney, M.J. Branch, H.S. Dua, A. Hopkinson, Effect of culture medium on propagation and phenotype of corneal stroma-derived stem cells, Cytotherapy 17(12) (2015) 1706-1722.

[28] J.V. Jester, A. Budge, S. Fisher, J. Huang, Corneal keratocytes: phenotypic and species differences in abundant protein expression and in vitro light-scattering, Investigative ophthalmology \& visual science 46(7) (2005) 2369.

[29] K. Musselmann, B. Alexandrou, B. Kane, J.R. Hassell, Maintenance of the keratocyte phenotype during cell proliferation stimulated by insulin, Journal of Biological Chemistry 280(38) (2005) 32634-32639.

[30] G. Perrella, P. Brusini, R. Spelat, P. Hossain, A. Hopkinson, H.S. Dua, Expression of haematopoietic stem cell markers, CD133 and CD34 on human corneal keratocytes, The British journal of ophthalmology 91(1) (2007) 94-9.

[31] A. Joseph, P. Hossain, S. Jham, R.E. Jones, P. Tighe, R.S. McIntosh, H.S. Dua, Expression of CD34 and L-selectin on human corneal keratocytes, Invest Ophthalmol Vis Sci 44(11) (2003) 4689-92.

[32] Y. Pei, R.Y. Reins, A.M. McDermott, Aldehyde dehydrogenase (ALDH) 3A1 expression by the human keratocyte and its repair phenotypes, Experimental eye research 83(5) (2006) 1063-1073.

[33] Y. Pei, D.M. Sherry, A.M. McDermott, Thy-1 distinguishes human corneal fibroblasts and myofibroblasts from keratocytes, Experimental eye research 79(5) (2004) 705-712.

[34] J.V. Jester, W.M. Petroll, P.A. Barry, H.D. Cavanagh, Expression of alpha-smooth muscle (alpha-SM) actin during corneal stromal wound healing, Investigative ophthalmology \& visual science 36(5) (1995) 809-819.

[35] S. Masur, J. Cheung, S. Antohi, Identification of integrins in cultured corneal fibroblasts and in isolated keratocytes, Investigative ophthalmology \& visual science 34(9) (1993) 2690-2698.

[36] C.E. Ghezzi, B. Marelli, F.G. Omenetto, J.L. Funderburgh, D.L. Kaplan, 3D Functional Corneal Stromal Tissue Equivalent Based on Corneal Stromal Stem Cells and Multi-Layered Silk Film Architecture, PLoS One 12(1) (2017) e0169504. 